\title{
Pooled Blood Products
}

National Cancer Institute

\section{Source}

National Cancer Institute. Pooled Blood Products. NCI Thesaurus. Code C133279.

Individual components, e.g. cryoprecipitated antihemophilic factor (AHF), whole bloodderived platelet concentrates, that are combined using an aseptic technique. 